This document is the accepted manuscript version of the following article:

Stravs, M. A., Pomati, F., \& Hollender, J. (2019). Biodiversity drives micropollutant biotransformation in freshwater phytoplankton assemblages. Environmental science and

Technology, 53(8), 4265-4273. https://doi.org/10.1021/acs.est.8b07018

\title{
1 Biodiversity drives micropollutant 2 biotransformation in freshwater phytoplankton assemblages
}

Michael A. Stravs ${ }^{\dagger \ddagger^{*}}$, Francesco Pomati†§, Juliane Hollender ${ }^{\dagger \ddagger^{*}}$

tEawag, Swiss Federal Institute of Aquatic Science and Technology, Überlandstrasse 133, 8600 Dübendorf, Switzerland

‡Institute of Biogeochemistry and Pollutant Dynamics, Universitätstrasse 16, ETH Zürich, 8092 Zürich, Switzerland

§Institute of Integrative Biology, ETH Zürich, Universitätstrasse 16, 8092 Zürich, Switzerland

*Corresponding Author, michael.stravs@eawag.ch, juliane.hollender@eawag.ch
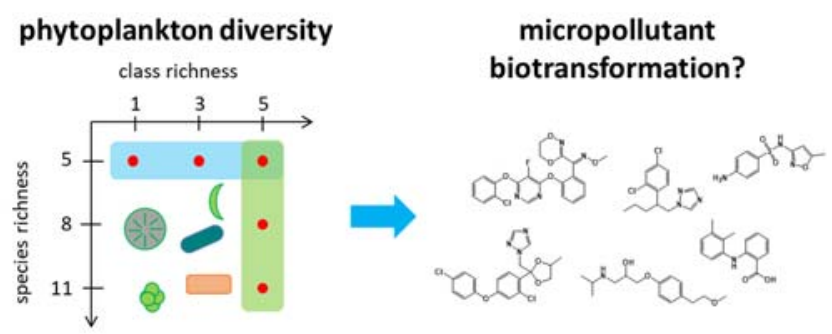

\section{Abstract}

Biotransformation of chemical pollutants is an ecological process requiring multifunctionality (multiple metabolic pathways) and, potentially, high biodiversity. Phytoplankton communities are highly diverse functionally and taxonomically, and co-occur with complex mixtures of organic pollutants in aquatic environments. Here, we investigated how phytoplankton species richness (SR) and class richness (CR) determine the biotransformation of a mixture of 37 structurally diverse pollutants using laboratory experiments and analysis of high-resolution mass-spectrometry data. Laboratory phytoplankton communities were assembled from pure cultures by creating a gradient from one to five taxonomic groups, and 5 to 11 total species, in defined medium. The biotransformation of pollutants over 6 days and the total number of transformed chemicals increased with CR for 13 considerably transformed compounds. The total number of transformation products (TPs, up to 42) was positively affected by both CR and SR: CR had a positive effect on stable TPs found, SR led to more transient TPs. Our data indicate that both taxonomic and functional diversity are important for biotransformation of anthropogenic chemicals in phytoplankton and suggest that plankton biodiversity could play a role in the remediation of pollutant loads in aquatic ecosystems. 
The environmental fate of anthropogenic chemical pollutants is of preeminent research interest as they can affect flora and fauna, and ultimately also human health. Surface waters receive inputs of organic pollutants from agricultural sources, e.g., pesticides, as well as from urban areas, e.g., pharmaceuticals and personal care products, which are not degraded in wastewater treatment plants. The fate of these micropollutants in aquatic ecosystems is governed by sorption to abiotic material, abiotic transformation, bioconcentration in organisms, and biotransformation. Biotransformation processes can lead to the complete degradation of a compound to $\mathrm{CO}_{2}$ (mineralization) or to the formation of transformation products (TPS). The latter can have properties (persistence, toxicity, sorption potential) distinct from their parent compounds, and the study of biotransformation is essential for understanding the environmental fate of micropollutants ${ }^{1,2}$.

Phytoplankton are primary producers at the basis of aquatic food-webs and have recently been studied as a potential driver of biotransformation processes. Thomas and Hand presented evidence that degradation of different micropollutants is influenced by the presence of phytoplankton ${ }^{3}$ and showed, for example, that a number of cyanobacteria and green microalgae are competent in the degradation of the fungicide fludioxonil ${ }^{4}$. Further, the biotransformation of selected estrogens, industrial chemicals, and herbicides by phytoplankton has been investigated in different studies ${ }^{5-8}$. We recently elucidated the transformation of 24 micropollutants by two cyanobacterial species and the green alga Chlamydomonas reinhardtii. For 10 micropollutants, we could observe 14 transformation products formed by oxidation, reduction, and conjugation reactions ${ }^{9}$. Phytoplankton is however a highly diverse, polyphyletic group of organisms, spanning the domains of bacteria and eukaryotes, and within the latter, distributed across different phyla ${ }^{10}$. Major groups are the Cyanobacteria (prokaryotic), Chlorophyta, Chrysophyta, Cryptophyta, and the Bacillariophyta (or diatoms; all eukaryotic). Whereas heterotrophic nutrition is observed in isolated cases for all listed groups, mixotrophy is common only in chrysophytes and cryptophytes ${ }^{11}$. The community composition of phytoplankton is very dynamic and continuously driven by fluctuations in abiotic and biotic variables ${ }^{12,13}$.

Given the breadth of organisms that make up the phytoplankton, it is of immediate interest how biotransformation processes of micropollutants in aquatic ecosystems might depend on phytoplankton community diversity and composition. Higher biodiversity is generally agreed to improve efficiency and stability in performed community functions and can contribute to enhanced provision of services ${ }^{14}$. The relationship between diversity and ecosystem functioning is however not trivial. Recent analyses suggest a saturating relationship, as at high diversity new species introduce increasingly redundant functions (but can contribute to stability) ${ }^{14}$. When multiple functions or services are considered, the influence of biodiversity is larger, i.e., higher biodiversity is required to provide multiple functions or services simultaneously (multifunctionality) ${ }^{14,15}$. In phytoplankton communities, species richness (SR) and trait diversity have been shown to improve the productivity and resource use efficiency in observational studies ${ }^{16,17}$. Regarding micropollutant degradation, a positive effect of bacterial diversity was observed in wastewater treatment plants on degradation rates of individual micropollutants, with the highest effect seen for multifunctionality (i.e., the composite biotransformation of multiple micropollutants) ${ }^{18}$.

Ecosystem effects are mediated by functions performed by assemblages of organisms and by the diversity of species and functional groups within; in many cases, the richness of broad taxonomic groups, like classes, which represents the diversity of phylogenetically conserved functional traits (e.g. metabolic pathways, pigments), is a more powerful measure than SR for 
species into classes (e.g., green algae, cyanobacteria) allows accounting for important ecological, physiological and genetic differences among phytoplankton species ${ }^{12,20,21}$. Here, we assume that phytoplankton classes as defined above differ in the physiological and genetic basis that reflect functional diversity for co-metabolic or metabolic biotransformation of micropollutants. Therefore, we hypothesize that both class richness (CR) and independently SR can influence biotransformation processes ${ }^{22}$.

This paper aims to test these hypotheses. To this end, we conducted experiments to determine the biotransformation potential of assembled communities of different CR (1-5 groups) and SR (5-11 species) on a mixture of 37 environmentally relevant micropollutants with a wide range of chemical structural features. Transformation of compounds as well as formation of TPs was assessed using liquid chromatography coupled to high-resolution mass spectrometry.

\section{Materials and Methods}

\section{Chemical mixture}

A mixture of 37 compounds (16 pharmaceuticals, 10 fungicides, 6 insecticides, and 4 additional chemicals) with a wide range of chemical structural features (therefore, potentially subject to different transformation pathways), molecular weight (120 to $792 \mathrm{Da}$ ), and hydrophobicity (log $\mathrm{K}_{\mathrm{ow}}-2.5$ to 5.8 ) was prepared from stock solutions of analytical grade $(95 \%+)$ reference standards (Table S3). Chemicals were obtained from Sigma-Aldrich, Fluka (now Sigma-Aldrich), Dr. Ehrenstorfer (now LGC Standards), TRC Canada, Lipomed AG, or Riedel-de Haën (see Table S3). In preliminary tests (see SI S1.1 and Figure S2), the mixture did not inhibit growth at a concentration of $2.5 \mu \mathrm{g} / \mathrm{mL}$ for one species per taxonomic class.

\section{Growth conditions}

Twenty-two cultures from five algal taxonomic classes were obtained from different sources (see Table S2) and were maintained in ca. $50 \mathrm{~mL}$ volume in Woods Hole Combo medium (WC), WC with added silicate $(\mathrm{WC}+\mathrm{Si})$ or WC with added heat-killed bacteria (WC+Bac; details see Supporting Information (SI)) in 100-mL Erlenmeyer flasks under a $8 \mathrm{~h} / 16 \mathrm{~h}$ day/night cycle in a temperature-controlled room at $20^{\circ} \mathrm{C}$. Sub-culturing occurred under sterile conditions (laminar flow cabinet) every 1 to 4 weeks depending on growth.

\section{Experiment}

Twenty-two algal species, belonging to one of 5 taxonomic classes (Chlorophyceans $\mathrm{CHL}$, Cyanobacteria CYA, Chrysophytes CHR, Cryptophytes CRY, and diatoms DIA) were precultured as described above. These species were arranged into 27 communities with different CR and SR: one experimental block with constant SR (SR 5) but varying CR (CR 1, 3 or 5) and one experimental block with constant CR (all classes present, CR 5) and varying SR (SR 5, 8 or 11; see Table S1 for experimental design). Five communities per SR/CR combinations were drawn. The SR 5 / CR 5 combination was shared between the experimental blocks. Note that the 5 communities for the special case SR 5 / CR 1 consisted in all the available species for the respective taxonomic group. While a number of cultures were examined, 5 species that grew in the selected medium could not be found for all classes. Therefore, only 3 species for CHR and 4 species for CRY could be used. In addition, a selection of SR 3 was performed for the classes $\mathrm{CHL}$ and CYA. The random selections were adjusted such that all taxonomic groups were evenly represented in SR 5 / CR 3 experiments 
and such that overall species representation was approximately even. All selections are listed in Table S4.

One week before the experiment, an aliquot of each species was diluted with fresh medium and incubated in an incubation shaker (Multitron II, Infors $\mathrm{HT}$ ) at $20^{\circ} \mathrm{C}, 90 \mathrm{rpm}$, and approximately $100 \mu$ Einstein light irradiation from fluorescent tubes. The fluorescent tubes were shielded by UV protection tubes (METOLIGHT ASR-UV-400-60-T8, Asmetec), and UV protection foil (METOLIGHT SFC-10, Asmetec) was used to cover the shaker window, to reduce possible photodegradation of chemicals by UV light during the subsequent experiment.

Before the experiment, the biovolume of each strain was determined from flow cytometry measurements of single cultures (see $\mathrm{SI}$ ) using a formula for biovolume estimation from Total Forward Scattering per particle ${ }^{23,24}$. For each selection, volumes of different species were calculated such that the total biovolume of all species combined was constant $\left(3.3 \times 10^{5} \mu \mathrm{g} / \mathrm{L}\right)$. Species evenness was not necessarily constant, since not all species were available in the same density; in isolated cases, the total biovolume could not be attained from the present culture densities. Biovolumes for each culture are listed in Table S5. For each selection, the specified volumes of each species were combined into a sterilized $20-\mathrm{mL}$ glass vial and adjusted with WC+0.5Si medium to a total volume of $6 \mathrm{~mL}$. Additionally, the 5 "CR 1/SR 5" selections were prepared in duplicate for use as chemical-negative controls (see below). In addition, two bacterial control selections were prepared: a constant volume of all $\mathrm{CHL}$ and CYA species except Synechococus (9 total, BAC 1) and all CHR, CRY, and DIA species (12 total, BAC 2) was pooled and filtered through a $1 \mu \mathrm{m}$ track-etched polycarbonate filter (Whatman Nuclepore, cat. no. 111110, Sigma-Aldrich). The filtrate, which contained only particles $<1 \mu \mathrm{m}$, represented the bacterial contaminations of all species. Because of their small size, Synechococcus was excluded from this control mixture. The filtrate was collected and adjusted with $\mathrm{WC}+0.5 \mathrm{Si}$ to $6 \mathrm{~mL}$. Two medium controls of WC+0.5Si were also prepared. This resulted in 36 total samples $(5 \times 5+2$ selections, 5 chemical-negative controls, 2 bacterial controls, 2 medium controls.) The vials were covered loosely with a plastic cover and incubated as above.

After 1 day of acclimation, $100 \mu \mathrm{L}$ of spike solution $(150 \mu \mathrm{g} / \mathrm{L}$ per compound in $7.5 \% \mathrm{EtOH} /$ $\mathrm{H}_{2} \mathrm{O}$ ) was added to each vial except the chemical-negative controls to a final concentration of $2.5 \mu \mathrm{g} / \mathrm{L}$ for each compound. To the chemical-negative controls, $100 \mu \mathrm{L} 7.5 \% \mathrm{EtOH}$ in $\mathrm{H}_{2} \mathrm{O}$ was added. Immediately, samples were withdrawn for chemical analysis and growth determination: $200 \mu \mathrm{L}$ were sampled into a 96-well plate and the optical density at $750 \mathrm{~nm}$ $\left(\mathrm{OD}_{750}\right)$ was determined (Cytation 5, Biotek). $550 \mu \mathrm{L}$ were diluted 1:1 with $\mathrm{MeOH}$ in $2 \mathrm{~mL}$ HPLC vials, mixed well, and incubated in an ultrasonic bath for 10 minutes at $37^{\circ} \mathrm{C}$. Subsequently, $350 \mu \mathrm{L}$ were transferred into flat-bottom glass inserts (SUPELCO, cat. no. 29441-U, Sigma-Aldrich) in Eppendorf tubes and centrifuged for $4 \mathrm{~min}$ at $9000 \mathrm{rpm}$. The supernatant was recovered into a $2 \mathrm{~mL} \mathrm{HPLC}$ vial and stored at $-20^{\circ} \mathrm{C}$ until analysis. After the initial (t0) sampling, the experimental vials were incubated as above. After 1, 2, 4, and 6 days, samples for chemical analysis and growth determination were taken as described above. The entire experiment was repeated after two weeks for replication, and both experiments were analyzed in common. Additionally, a sample was taken at 4 days (first replicate experiment) and 6 days (second replicate experiment) for bacterial counting by flow cytometry. $500 \mu \mathrm{L}$ were sampled into a $2 \mathrm{~mL}$ HPLC vial, and fixed with $0.01 \%$ paraformaldehyde and $0.1 \%$ glutaraldehyde. These samples were stored at $4^{\circ} \mathrm{C}$ until measurement.

\section{Chemical analysis}

Compound concentrations were determined using online solid phase extraction coupled to LCHRMS based on a previously published method ${ }^{9}$. Briefly: $150 \mu \mathrm{L}$ of supernatant sample was 
diluted to $20 \mathrm{~mL}$ with nanopure water and fortified with internal standard (IS) solution (absolute quantity $187.5 \mathrm{pg}$ per IS compound per sample). The $20 \mathrm{~mL}$ sample was enriched on a custom multilayer online SPE cartridge (see SI) and eluted with $\mathrm{MeOH} / 0.1 \%$ formic acid (FA) onto the analytical column (Atlantis T3, $3 \mu \mathrm{m}, 150 \mathrm{~mm}$ ) after predilution with $\mathrm{H}_{2} \mathrm{O} / 0.1 \% \mathrm{FA}$. Chromatography was performed with a 13.3-95\% MeOH / 0.1\% FA in $\mathrm{H}_{2} \mathrm{O} / 0.1 \%$ FA gradient over $32 \mathrm{~min}$. A quadrupole-Orbitrap mass spectrometer (Q-Exactive, Thermo Scientific) with a heated-electrospray source was used for detection. Measurements were performed in MS1 and data-independent MS2 in polarity switching mode. Analytes were quantified using the internal standard method with TraceFinder EFS (version 3.2.368.22, Thermo Scientific). Details are listed in the SI. The raw calculated amounts were exported in csv format.

\section{Transformation product screening}

For 13 compounds with quantifiable biotransformation a list of potential TPs was generated. Using the open-source workflow RMassScreening (https://github.org/meowcat/ RMassScreening), the sample time series were screened for potential TPs occurring in culture samples, not in bacterial controls, chemical-negative controls, or the $t_{0}$ samples. Details are listed in the SI.

\section{Data evaluation and transformation assessment}

All data processing and statistical evaluation was performed in R. Three compounds were persistent in all samples (thiamethoxam, hydrochlorothiazide, sucralose), and all concentrations were divided by the mean of these compounds to correct for evaporation in the samples, sampling inaccuracies, etc. This resulted in a time series for 37 compounds in 72 experiments. Values for two missing samples (out of 288 total) were imputed from the preceding time point. Exemplar time series plots are shown in Fig. S3.

For each compound, two measures of the transformation rate were fitted to each time series (transformation integral \%deg and transformation rate $\mathrm{k}$ ). The transformation integral \%deg was determined as the area under the curve of the relative amount of compound removed since the start of the experiment:

$$
\% \operatorname{deg}=\frac{1}{6 d C_{0}}\left(\int_{t=0}^{6 d} C_{o}-C(t)\right)
$$

as described in SI S1.3 and illustrated in Fig. S1. This resulted in a value roughly in the $[0,1]$ range where 0 means no transformation and 1 means total transformation. The transformation rate $\mathrm{k}\left({\left.\text { in } \mathrm{d}^{-1}\right)}\right.$ ) was determined by nonlinear fitting of the equation $C=C_{0} e^{-k t}$, where $\mathrm{C}_{0}$ was set as the mean of all $t_{0}$ concentrations for the compound. $k$ is a value roughly in the $[0$,infinity] range where 0 means no transformation (and 1 corresponds to one natural logarithm unit attenuation per day). Not all compounds showed transformation trends that qualitatively fit firstorder kinetics, likely because of community dynamics effects; notwithstanding, the obtained values for $k$ qualitatively appear to describe the extent and speed of transformation well.

In Fig. S4, \%deg and k corresponded well for all compounds, and the relationship was linear except for rare samples for which transformation integrals were near 1 , since they cannot capture differences in extremely fast transformation processes. (e.g., for kresoxim-methyl). For further analysis, compounds were classified as "transforming" when they showed a maximum $\%$ deg of 0.2 and above, and significant number of samples with transformation stronger than the bacterial control (see SI). The bacterial control was preferred over medium control since it reflects sample conditions closer; in addition, medium controls without biomass appeared susceptible to abiotic transformation, possibly through indirect photolysis. 
$\mathrm{OD}_{750}$ minus background was used as a proxy to correct for biomass effects. All \%deg and $\mathrm{k}$ values were divided by the biomass integral for each sample, computed from $\mathrm{OD}_{750}$ in analogy to \%deg (see SI). For each compound, the corrected rates were centered to zero mean and scaled to unit standard deviation to examine diversity effects on each compound equally, independent of the total average transformation rate of the compound.

\section{Statistical analysis}

The number of transformed compounds (\#TC) was computed for each sample. For \#TC determination, biomass-corrected \%deg or $\mathrm{k}$ were scaled to a maximum of 1 , where 0 was the maximal transformation rate observed in a control sample and 1 the maximal overall transformation rate. For each sample, the number of substances exceeding a cutoff relative to the maximal transformation rate were counted.

The micropollutant multifunctionality (MPMF) was computed as proposed by Johnson et al. ${ }^{18}$. For each compound, transformation rates were centered and scaled to a mean of zero and a standard deviation of one. For each sample, the normalized transformation rates for all compounds were averaged.

The influence of $\mathrm{CR}$ and $\mathrm{SR}$ on individual compound transformation, \#TC, MPMF, or biotransformation products observed was determined with a one-sided Pearson correlation test of $C R$ or $S R$, respectively, to the examined value.

For visualization of compound correlations, the Pearson correlation matrix of $\mathrm{k}$ or \%deg values was ordered by hierarchical clustering using Euclidean distances and complete cluster linkage.

\section{Results and Discussion} \\ Overall extent of transformation}

Of the 37 tested compounds, 13 showed measureable transformation in polyculture samples: atenolol, azoxystrobin, benzotriazole, carbendazim, climbazole, cyprodinil, kresoxim-methyl, mefenamic acid, metoprolol, tebuconazole, venlafaxine, fipronil and fludioxonil (see Table S8). Transformation integrals for these compounds ranged from partial (\%deg 0.2 , or $k=0.06 \mathrm{~d}^{-1}$ for venlafaxine) to complete (\%deg 1.09 , or $\mathrm{k}=1.37 \mathrm{~d}^{-1}$ for atenolol). From the remaining compounds, sulfamethoxazole also showed fast transformation in a number of samples, but was degraded in one bacterial control (no phytoplankton, BAC 2) for both experimental replicates and therefore discarded from further evaluation.

Thirteen compounds were stable, i.e., were not transformed in any sample, and one (methoxyfenozide) degraded only in medium controls (no phytoplankton or bacteria), possibly by indirect photolysis. For the remaining 9 compounds, quantification was inaccurate because of interferences or too low concentrations; higher concentrations were not used to avoid toxicity. These compounds were not further evaluated, and only a tentative classification of the transformation behaviour was given. Details for all 37 compounds are shown in Table S8. Influence of class and species richness on single compound transformation

In Fig. 1, the biomass corrected and scaled biotransformation integrals (\%deg, see Methods) are shown separated by $\mathrm{CR}$ and SR for each of the 13 compounds with quantifiable biotransformation. On top, the Pearson correlation coefficients are shown for each compound for \%deg against $C R$ in all $S R \leq 5$ experiments (influence of $C R, n=34$ for each compound), and against $S R$ in all $C R=5$ experiments (influence of $S R, n=30$ for each compound). The overall effect is given by the distribution of $C R$ and $S R$ effects on each compound ( $n=13)$. Fig. S5 shows the identical metrics calculated on the transformation rate $\mathrm{k}$. 
As a general trend, biodiversity had a positive influence on biotransformation (Fig. 1). The influence of CR is significant for both \%deg and k (\%deg: $p<0.001 ; k: p=0.015, n=13$ ), whereas the SR effect is not significant for either measure (\%deg: $p=0.25, k: p=0.35, n=13$ ). It is apparent that different compounds show different trends in their transformation patterns. For example, the transformation of benzotriazole and climbazole depends strongly on the CR but not on SR; whereas for azoxystrobin, imidacloprid, or kresoxim-methyl, transformation is more strongly related to SR and hardly affected by CR. For all compounds except atenolol, either CR, SR, or both effects were positive. If only statistically significant slopes $(p<0.05$, one-sided Pearson correlation test) for each compound are considered, then 4 positive CR effects are observed for \%deg (3 positive effects for $\mathrm{k}$ ) and no significant SR effect is found.

The same analysis was conducted without biomass correction. For this case, the CR effect is significant only for \%deg $(p=0.04)$ but not for $k(p=0.27$, both $n=13)$. This is because of a specific $\mathrm{CR}=1$ experiment ( $\mathrm{CHR}$ class), which exhibited fast growth and concomitantly fast transformation of multiple compounds (carbendazim, azoxystrobin, cyprodinil, kresoximmethyl), reducing the net $\mathrm{CR}$ effect as a result. As expected, a model using robust linear regression excludes this effect and gives a stable relationship ( $p=0.009$ for \%deg, 0.03 for $k$, $n=13$.)

\section{Influence of class and species richness on overall compound transformation}

To complement these results, the biotransformation potential of communities was also assessed as the number of transformed compounds (\#TC). The cutoff value for compound transformation was determined to maximize the range of \#TC values over the cultures. We calculated micropollutant multifunctionality (MPMF) using centered, scaled rates of change for all compounds that were averaged per experiment, such that the resulting average represents a normalized relative biotransformation potential for each community, as suggested by Johnson et al. ${ }^{18}$. For both \#TC and MPMF, significantly positive effects of CR and nonsignificant effects of SR were found when analyzed with either transformation integrals (Fig. 2) or rates (Fig. S6).

This suggests that the overall transformation potential of a phytoplankton community is mainly dependent on the number of taxonomic classes that can potentially be active; whereas once all taxonomic groups are present, additional species have a small effect. This would correspond to what is expected when the metabolic / genetic basis for biotransformation are similar within taxonomic groups. In this case, the diversity of enzymes potentially active in transformation is, as a first approximation, determined by the identity or total number of taxonomic classes present.

Further analyses were conducted to verify that the observed effects of $C R$, both for single compounds and overall transformation, are indeed caused by overall diversity rather than a single taxonomic class (sampling effect; SI S2.5, Tables S9-11). In summary, it was found that for some compounds individual taxonomic classes were significantly important, but that no single group dominated the total community effect. Notably, diatoms presence showed only negative effects, possibly due to slow growth of these strains in the communities.

Finally, to verify that bacterial growth did not influence the results, bacterial counts were assessed after 6 days (first experiment) or 4 days (second experiment) using staining and flow cytometry. All corresponding gated samples are shown in Figure S10. The results showed that bacterial growth was minor; in fact, most counts in the gate corresponding to bacteria can be attributed to cyanobacteria that are not clearly separated from bacteria but have their center outside the counted gate. From this, we conclude that bacterial growth was not a driver behind biotransformation in the experiment. 


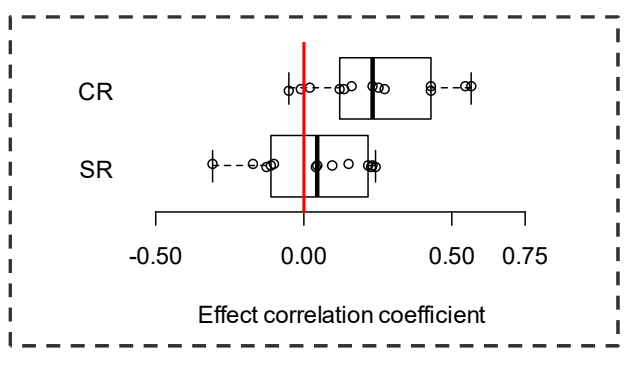

CR effect

$\mathrm{CR}=1$ to 5

$\mathrm{SR}=5$

SR effect

$\mathrm{CR}=5$

$\mathrm{SR}=5$ to 11
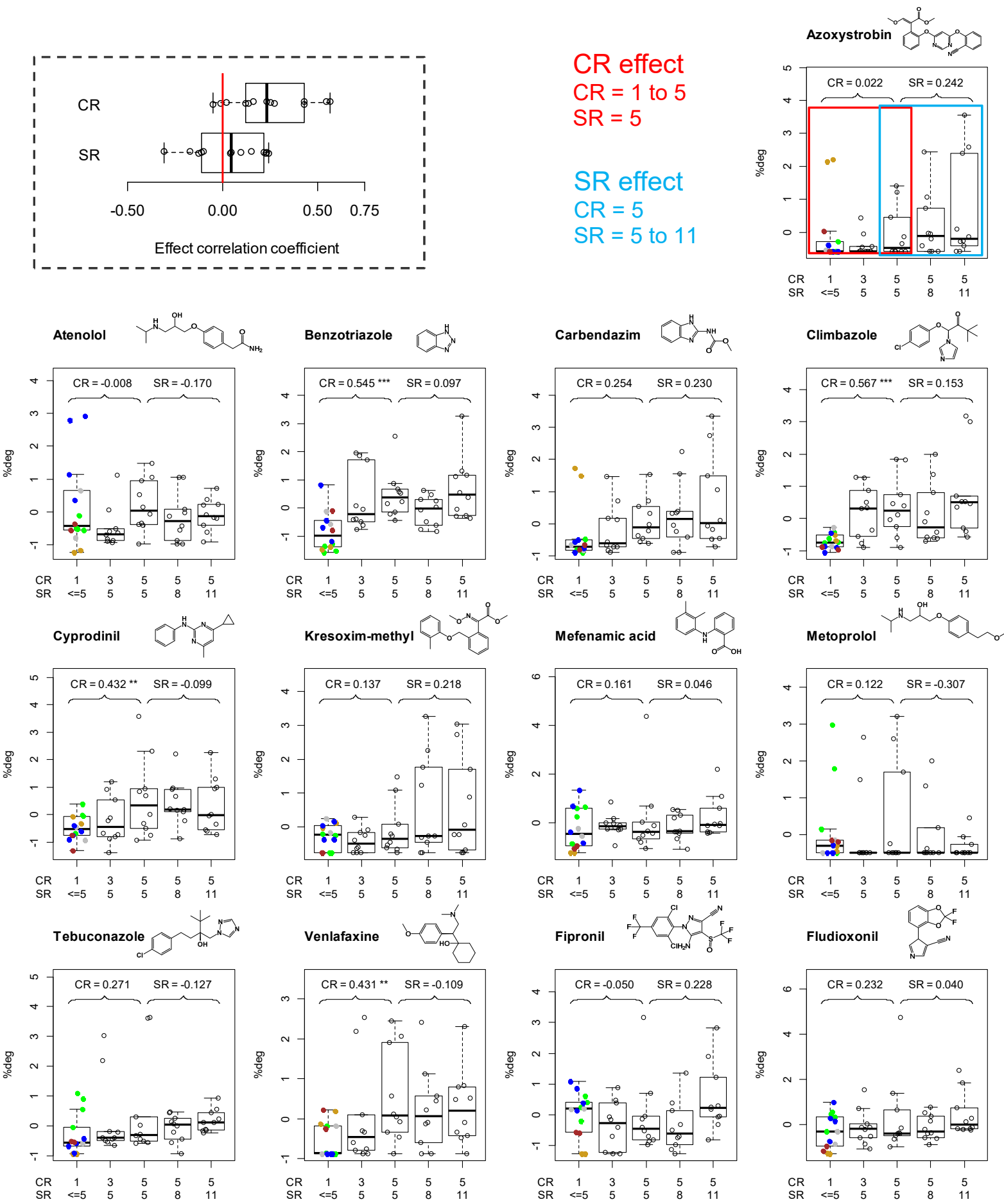

Figure 1. Top left: Distribution of class richness (CR) effect and species richness (SR) effect slopes for all compounds for \%deg values. Red line indicates the zero effect line. Top right and below: Distribution of \%deg values, for each compound, separated by CR and SR, and Pearson correlation coefficients for CR and SR effects (top right, illustrated example for azoxystrobin). On top Pearson correlation coefficient for CR and SR effects, respectively. *: $p<0.05 ;{ }^{* *}: p<0.01 ;{ }^{* * *}: p<0.001$. For $C R=1$, colors indicate the class: green, chlorophytes; blue, cyanobacteria; golden, chrysophytes; brown, diatoms; grey, cryptomonads. Boxplots represent median and first/third quartile (hinges) and the most extreme data points no more than $1.5 \mathrm{x}$ the interquartile range from the box (whiskers). 

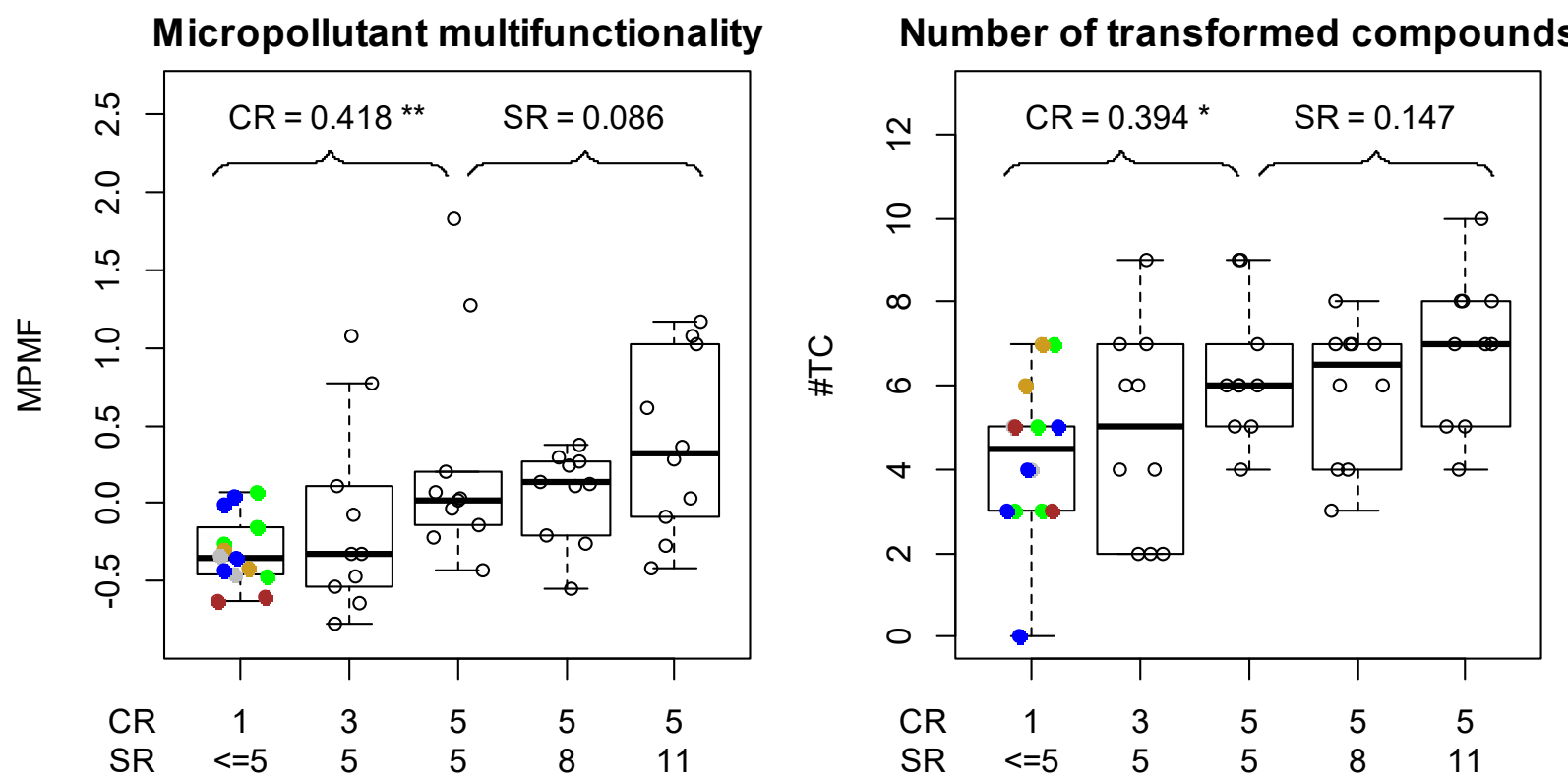

Figure 2. Influence of $\mathrm{CR}$ and SR on micropollutant multifunctionality (MPMF, left) and number of transformed compounds (\#TC, right). On top Pearson correlation coefficient for CR and SR effects, respectively. ${ }^{*}: p<0.05 ;{ }^{* *}: p<0.01 ;{ }^{* * *}: p<0.001$. Colors for taxonomic groups, and boxplot margins are as specified in Fig. 1. 
328 Given that the biotransformation of each compound was influenced by different classes, we further inquired whether there are groups of similarly behaving compounds, whose transformation is performed by similar groups of organisms. Heat maps (Fig. 3, Fig. S7) visualize the correlation matrix of all \%deg and $k$ values, respectively, across all selections by compounds, ordered by hierarchical clustering. For each compound, the effects of CR and SR, and of individual taxonomic classes presence/absence are also shown, to identify common factors driving the transformation behavior. Considering either $\mathrm{k}$ or \%deg, three main groups are apparent: The two strobilurin fungicides (kresoxim-methyl and azoxystrobin) and carbendazim fall into group 1, venlafaxine and metoprolol, both rather polar with larger alipathic moieties, build group 2, and a larger cluster with multiple compounds with nitrogen heterocycles (climbazole, tebuconazole, benzotriazole, fipronil, mefenamic acid, and fludioxonil) belong to group 3. Atenolol shows weak correlation to any other compound. Cyprodinil is assigned to group 3, but also shows similarity to group 1. Overall, strongly correlated groups are noted, which are often associated with specific chemical moieties, but little apparent anticorrelation, suggesting that no evident tradeoffs exist between transformations of different compounds.

Fig. 3 (bottom) shows the compound groups with chemical structures and associated CR, SR, and composition effects. Group 1 is characterized by positive (though non-significant) effects of SR, positive effects of CHR, and negative effects of CRY. Group 2 is associated with positive CR effects and negative SR effects, as well as positive CHR and negative CRY effects. For the broad group 3, a CR effect can be observed best since both CR and multiple individual classes show a positive influence. Whereas these interpretations have anecdotal character, they suggest that transformation mechanisms for different structural features might be unequally common, and differently distributed within algal classes.

\section{Influence of taxonomic group and species richness on transformation products}

TPs observed for micropollutants reflect metabolic pathways active in their respective community, but their relationship with community diversity is non-trivial. On one hand, a higher richness suggests the presence of more transformation pathways, which should lead to a higher variety of observed TPs. On the other hand, high richness can also lead to first-order TPs being (quickly) further transformed into structurally more distant, very small or polar TPs, or even mineralized. This would lead to less apparent/observable TPs (in particular firstgeneration TPs) detectable with our LC-HRMS analysis. To shed light on this relationship, the entire dataset was screened for masses of $>1000$ putative TPs of selected compounds for which notable transformation in the assembled communities was observed. The search was limited to expected first-generation TPs and extended to later generations of observed firstgeneration products (see $\mathrm{SI}$ ). Whereas the identified chemical features are reasonably likely to be TPs, detailed structure elucidation and confirmation was outside the scope of this study. Forty two TPs were observed in total. Thereof, 15 were attributed to the parent fludioxonil, 12 to metoprolol, and 5 to cyprodinil, whereas 1 to 3 TPs could be found for the remaining compounds. The observed atenolol acid is a well-known TP of both atenolol and metoprolol. To facilitate analysis, it was attributed to atenolol alone. No TPs were observed for benzotriazole, climbazole and venlafaxine. 

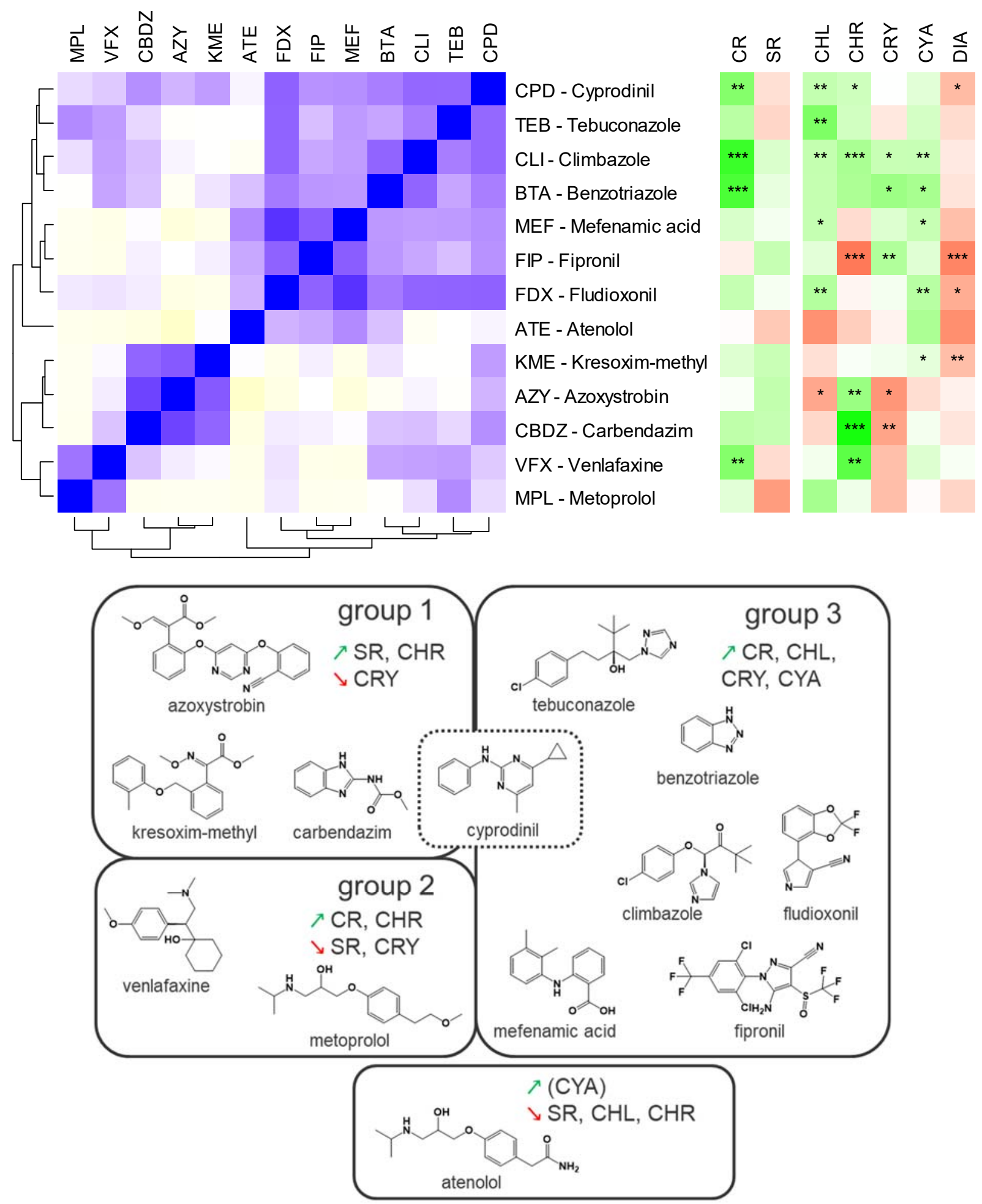

Figure 3. Top left: Hierachical clustering of Pearson correlation coefficients between compound transformation integrals (\%deg) across all samples. Blue, positive correlation; white, no correlation; yellow, negative correlation. Column names are the compound name abbreviations as specified in rows. Top right: Effect of CR and SR (as from Fig. 1), and effect of presence/absence of individual taxonomic groups in $\mathrm{CR}=1$ and $\mathrm{CR}=3$ samples, on \%deg. Green, positive effect; white, no effect; red, negative effect. *: $p<0.05{ }^{* *}$ : $p<0.01 ;{ }^{* * *}: p<0.001$. Bottom: Compounds as grouped by hierarchical clustering. 
For each TP and experimental sample, the integral of peak area (total peak area) under the linearly interpolated time trend $\mathrm{C}(\mathrm{t})$ was calculated and corrected by biomass. Correlation coefficients for CR and SR influence were then calculated as above; the presence of the TPs was also checked in bacterial controls. Individually analyzed, most TPs show uncharacteristic behavior, since they appear only in certain samples (Fig. S9). However, for selected TPs, a clear trend can be observed, e.g. three putative fludioxonil TPs, four putative metoprolol TPs, and one mefenamic acid TP show a significant positive correlation with increasing CR.

When considering the entirety of TPs, a count of observed different TPs per sample shows a significant positive slope for CR and SR and a net overall positive effect (Fig. 4). TPs were then separated into "transient" and "stable": TPs were denoted "transient" when their final peak area was $<50 \%$ of their maximal peak area, indicating that they are being further transformed to another product. Notably, the count of stable TPs shows a significant positive slope for CR and no effect for SR, whereas the count of transient TPs shows no effect for CR and a significant positive slope for SR (Fig. 4).
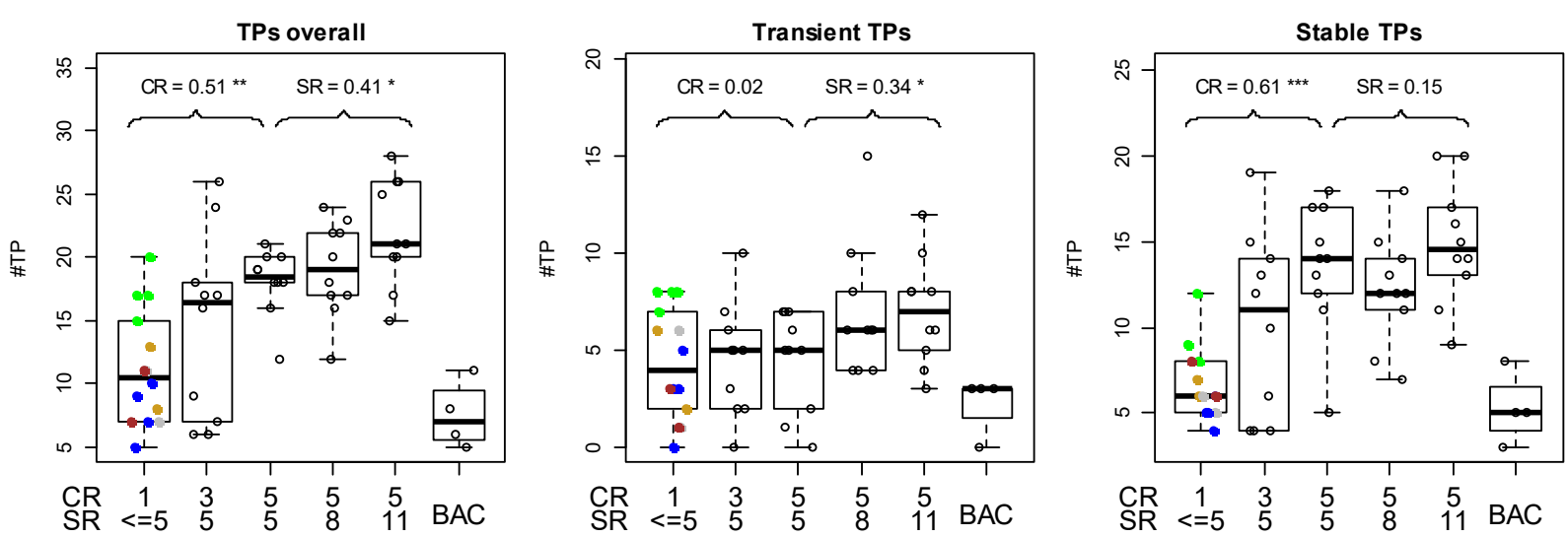

Figure 4. Number of observed transformation products dependent on CR and SR. Left, all TPs; middle, transient TPs (see text); right, stable TPs (see text). BAC, bacterial control. On top, Pearson correlation coefficient for $C R$ and $S R$ effects, respectively $(n=34$ and $n=30$ for $C R$ and SR effect, respectively.) *: $p<0.05 ;{ }^{* *}: p<0.01 ;{ }^{* * *}: p<0.001$. Colors for classes, and boxplot margins are as specified in Fig. 1.

The results for stable TPs reflect the general findings above and are expected in light of the observed positive relationship between CR and number of compounds transformed (\#TC, Fig. 2). By contrast, the positive effect of SR on transient TPs unravel novel results that were hidden in the parent transformation rate analysis. The increased number of transient TPs represents additional biotransformation mechanisms, leading to further modification of TPs, or formation of the same final TP via different intermediates. These additional biotransformation pathways do not result in enhanced overall transformation of the studied compounds, but instead could contribute to the transformation of other compounds beyond those investigated in this study.

In summary, our data support the hypothesis that increased biodiversity leads to more observable TPs. By contrast, a general trend towards "further biotransformation" (i.e., a shift towards structurally distant or highly polar transformation products not captured by screening, or mineralization), which should result in a negative influence of biodiversity on the number of stable TPs, is not supported by the data. However, trends matching "further biotransformation" can be observed for individual compounds including mefenamic acid and cyprodinil (see $\mathrm{SI}$, 
Fig. S8). These examples show that the consequences of phytoplankton biodiversity for micropollutants transformation can be more complex than just additive effects, and reinforce the need for additional future studies ${ }^{14}$.

\section{Environmental Significance}

424

425

426

427

428

429

430

431

432

433

434

435

436

437

438

439

440

441

442

443

444

445

446

447

448

449

450

451

452

453

454

To the best of our knowledge this study reports the first evidence for phytoplankton biodiversity and composition effects on micropollutant biotransformation (37 compounds). Our work highlights the need to address the effects of biodiversity on ecosystem processes, such as the degradation of pollutants, at a comprehensive level: a study on a single compound alone would not have provided the same detailed and convincing data as reported here. In this study, results were facilitated by modern high-resolution mass spectrometry, which can be used to quantify the transformation of a large number of compounds in parallel, and further allowed to investigate TP formation at the community-level.

In nature, the effects of phytoplankton community composition and richness on micropollutant biotransformation will be dependent on the complex interaction between these organisms and their fluctuating environment. For example, phytoplankton taxonomic groups have different abiotic and biotic environmental preferences and community composition follows environmental gradients over time and space (e.g., seasonal succession, trophic state of the aquatic ecosystem) ${ }^{12,13}$. Our results suggest that meso-oligotrophic environments, generally characterized by high functional and taxonomic diversity ${ }^{12,25}$, will perform better than eutrophic ecosystems in biotransforming micropollutants. Biotransformation can additionally be influenced by the total biomass, which peaks in summertime and in eutrophic ecosystems ${ }^{26}$. These instances, of which an example are cyanobacterial blooms, do not coincide with a high biodiversity of the planktonic community ${ }^{12,13}$.

All the above factors influence the importance of phytoplankton in relation to other environmental fate processes of micropollutants. Importantly, the present work is concerned with the contribution of phytoplankton alone. In natural systems, phytoplankton communities occur in a complex environment, where biodegradation by heterotrophic bacteria is often the dominant process. Total biotransformation may be driven by bacterial abundance and diversity, and phytoplankton might only make a smaller contribution. In addition, abiotic processes such as photodegradation contribute to the fate of various chemicals. Therefore, experiments in more complex systems, such as mesocosm or field studies, are required to understand the relevance of phytoplankton and its diversity in micropollutant fate.

\section{Data availability}

The data sets generated and analyzed for the current study are available from the corresponding authors upon reasonable request.

\section{Acknowledgements}

We thank M. K. Thomas (Eawag) for helpful suggestions in experimental design and data analysis. We thank C. B. Mansfeldt for helpful comments on the manuscript. For support in flow cytometry, we thank J. Sigrist and F. Hammes. This work was supported by the Swiss National Science Foundation, grant number 315230_141190 and 205320_165935. 


\section{Supporting Information Available}

Detailed materials and methods, tables of cultures and chemical compounds used, supplementary evaluations supporting the main findings. This information is available free of charge via the Internet at http://pubs.acs.org.

\section{References}

(1) Boxall, A. B.; Sinclair, C. J.; Fenner, K.; Kolpin, D.; Maund, S. J. When Synthetic Chemicals Degrade in the Environment. Environ. Sci. Technol. 2004, 38 (19), 368A$375 \mathrm{~A}$.

(2) Sinclair, C. J.; Boxall, A. B. A. Assessing the Ecotoxicity of Pesticide Transformation Products. Environ. Sci. Technol. 2003, 37 (20), 4617-4625.

(3) Thomas, K. A.; Hand, L. H. Assessing the Potential for Algae and Macrophytes to Degrade Crop Protection Products in Aquatic Ecosystems. Environ. Toxicol. Chem. 2011, 30 (3), 622-631. https://doi.org/10.1002/etc.412.

(4) Thomas, K. A.; Hand, L. H. Assessing the Metabolic Potential of Phototrophic Communities in Surface Water Environments: Fludioxonil as a Model Compound. Environ. Toxicol. Chem. 2012, 31 (9), 2138-2146. https://doi.org/10.1002/etc.1928.

(5) Subashchandrabose, S. R.; Ramakrishnan, B.; Megharaj, M.; Venkateswarlu, K.; Naidu, R. Mixotrophic Cyanobacteria and Microalgae as Distinctive Biological Agents for Organic Pollutant Degradation. Environ. Int. 2013, 51, 59-72. https://doi.org/10.1016/j.envint.2012.10.007.

(6) Della Greca, M.; Pinto, G.; Pistillo, P.; Pollio, A.; Previtera, L.; Temussi, F. Biotransformation of Ethinylestradiol by Microalgae. Chemosphere 2008, 70, 20472053. https://doi.org/10.1016/j.chemosphere.2007.09.011.

(7) Lai, K.; Scrimshaw, M.; Lester, J. Biotransformation and Bioconcentration of Steroid Estrogens by Chlorella Vulgaris. Appl. Environ. Microbiol. 2002, 68 (2), 859-864. https://doi.org/10.1128/AEM.68.2.859-864.2002.

(8) Maes, H. M.; Maletz, S. X.; Ratte, H. T.; Hollender, J.; Schae, A. Uptake, Elimination, and Biotransformation of $17 \alpha$-Ethinylestradiol by the Freshwater Alga Desmodesmus Subspicatus. Environ. Sci. Technol. 2014, 48 (20), 12354-12361. https://doi.org/10.1021/es503574z.

(9) Stravs, M. A.; Pomati, F.; Hollender, J. Exploring Micropollutant Biotransformation in Three Freshwater Phytoplankton Species. Environ. Sci. Process. Impacts 2017, 19 (6), 822-832. https://doi.org/10.1039/C7EM00100B.

(10) Falkowski, P. G.; Katz, M. E.; Knoll, A. H.; Quigg, A.; Raven, J. A.; Schofield, O.; Taylor, F. J. R. The Evolution of Modern Eukaryotic Phytoplankton. Science 2004, 305 (5682), 354-360. https://doi.org/10.1126/science.1095964.

(11) Bellinger, E. G.; Sigee, D. C. Introduction to Freshwater Algae. In Freshwater Algae; Bellinger, E. G., Sigee, D. C., Eds.; John Wiley \& Sons, Inc., 2015; pp 1-42. https://doi.org/10.1002/9781118917152.ch1.

(12) Reynolds, C. S.; Huszar, V.; Kruk, C.; Naselli-Flores, L.; Melo, S. Towards a Functional Classification of the Freshwater Phytoplankton. J. Plankton Res. 2002, 24 (5), 417-428. https://doi.org/10.1093/plankt/24.5.417.

(13) Sommer, U.; Adrian, R.; De Senerpont Domis, L.; Elser, J. J.; Gaedke, U.; Ibelings, B.; Jeppesen, E.; Lürling, M.; Molinero, J. C.; Mooij, W. M.; et al. Beyond the Plankton Ecology Group (PEG) Model: Mechanisms Driving Plankton Succession. Annu. Rev. Ecol. Evol. Syst. 2012, 43 (1), 429-448. https://doi.org/10.1146/annurev-ecolsys110411-160251.

(14) Cardinale, B. J.; Duffy, J. E.; Gonzalez, A.; Hooper, D. U.; Perrings, C.; Venail, P.; Narwani, A.; Mace, G. M.; Tilman, D.; Wardle, D. A.; et al. Biodiversity Loss and Its Impact on Humanity. Nature 2012, 486 (7401), 59-67. https://doi.org/10.1038/nature11148. 
(15) Zavaleta, E. S.; Pasari, J. R.; Hulvey, K. B.; Tilman, G. D. Sustaining Multiple Ecosystem Functions in Grassland Communities Requires Higher Biodiversity. Proc. Natl. Acad. Sci. 2010, 107 (4), 1443-1446. https://doi.org/10.1073/pnas.0906829107.

(16) Ptacnik, R.; Solimini, A. G.; Andersen, T.; Tamminen, T.; Brettum, P.; Lepistö, L.; Willén, E.; Rekolainen, S. Diversity Predicts Stability and Resource Use Efficiency in Natural Phytoplankton Communities. Proc. Natl. Acad. Sci. 2008, 105 (13), 5134-5138. https://doi.org/10.1073/pnas.0708328105.

(17) Fontana, S.; Thomas, M. K.; Moldoveanu, M.; Spaak, P.; Pomati, F. Individual-Level Trait Diversity Predicts Phytoplankton Community Properties Better than Species Richness or Evenness. ISME J. 2018, 12 (2), 356-366. https://doi.org/10.1038/ismej.2017.160.

(18) Johnson, D. R.; Helbling, D. E.; Lee, T. K.; Park, J.; Fenner, K.; Kohler, H. P. E.; Ackermann, M. Association of Biodiversity with the Rates of Micropollutant Biotransformations among Full-Scale Wastewater Treatment Plant Communities. Appl. Environ. Microbiol. 2015, 81 (2), 666-675. https://doi.org/10.1128/AEM.03286-14.

(19) Díaz, S.; Cabido, M. Vive La Différence: Plant Functional Diversity Matters to Ecosystem Processes. Trends Ecol. Evol. 2001, 16 (11), 646-655. https://doi.org/10.1016/S01695347(01)02283-2.

(20) Behl, S.; Donval, A.; Stibor, H. The Relative Importance of Species Diversity and Functional Group Diversity on Carbon Uptake in Phytoplankton Communities. Limnol. Oceanogr. 2011, 56 (2), 683-694. https://doi.org/10.4319/lo.2011.56.2.0683.

(21) Stockenreiter, M.; Haupt, F.; Graber, A.-K.; Seppälä, J.; Spilling, K.; Tamminen, T.; Stibor, H. Functional Group Richness: Implications of Biodiversity for Light Use and Lipid Yield in Microalgae. J. Phycol. 2013, 49 (5), 838-847. https://doi.org/10.1111/jpy.12092.

(22) Lefcheck, J. S.; Byrnes, J. E. K.; Isbell, F.; Gamfeldt, L.; Griffin, J. N.; Eisenhauer, N.; Hensel, M. J. S.; Hector, A.; Cardinale, B. J.; Duffy, J. E. Biodiversity Enhances Ecosystem Multifunctionality across Trophic Levels and Habitats. Nat. Commun. 2015, 6, 6936. https://doi.org/10.1038/ncomms7936.

(23) Pomati, F.; Nizzetto, L. Assessing Triclosan-Induced Ecological and Trans-Generational Effects in Natural Phytoplankton Communities: A Trait-Based Field Method. Ecotoxicology 2013, 22 (5), 779-794. https://doi.org/10.1007/s10646-013-1068-7.

(24) Foladori, P.; Quaranta, A.; Ziglio, G. Use of Silica Microspheres Having Refractive Index Similar to Bacteria for Conversion of Flow Cytometric Forward Light Scatter into Biovolume. Water Res. 2008, 42 (14), 3757-3766. https://doi.org/10.1016/j.watres.2008.06.026.

(25) Mittelbach, G. G.; Steiner, C. F.; Scheiner, S. M.; Gross, K. L.; Reynolds, H. L.; Waide, R. B.; Willig, M. R.; Dodson, S. I.; Gough, L. What Is the Observed Relationship Between Species Richness and Productivity? Ecology 2001, 82 (9), 2381-2396. https://doi.org/10.1890/0012-9658(2001)082[2381:WITORB]2.0.CO;2.

(26) Tao, Y.; Yu, J.; Lei, G.; Xue, B.; Zhang, F.; Yao, S. Indirect Influence of Eutrophication on Air - Water Exchange Fluxes, Sinking Fluxes, and Occurrence of Polycyclic Aromatic Hydrocarbons. Water Res. 2017, 122, 512-525. https://doi.org/10.1016/j.watres.2017.06.026. 